\title{
INTUMESCENCES
}

SOCIÉTÉ HydRotechNique dE France

15 juin 1961

\section{Influence de quelques paramètres sur une onde de crue subite à l'aval d'un barrage}

\section{The influence of certain parameters on a sudden flood wave downstream from a dam}

\author{
Professeur L. ESCANDE \\ MEMBRE DE L'INSTITUT \\ DIRECTEUT DES LABORATOIRES D'HYDRAULIQUUE \\ DE L'E.N.S.E.H. DE TOULOUSE \\ L. CASTEX \\ LAURÉAT DE L'INSTITU'T \\ INGÉNIEUR EN CHEF \\ DU LABORATOIRE D'HYDRAULIQUE DE BaNLÈVE
}

PAR

\author{
Professeur .J. NOUGARO \\ LAURÉAT DE L'INSTITUT \\ DIRECTEUR TECHNIOUE \\ A L'INSTITUT DE MÉCANique DES FLUIDES \\ H. BARTHET \\ IICENCIÉ ¿̀s SCIENCES \\ STAGIATRE DE REGHERCHES AU C.N.R.S.
}

ET

\begin{abstract}
Premiers résultats expérimentanx obtenus sur un modèle réduit représentant, à l'échelle de 1/300, la vallée de la Truyère à l'aval du barrage de Sarrans.

Célérité de l'onde, sa forme et son amplitude en fonction de différents paramètres: nivean dans la reterune amont, débit initial en aval du barrage, rugosité du lit de la rivière.
\end{abstract}

Initial experimental results obtained with a 1:300 scale model of the Truyere valletg below the Sarrans dam.

Wave celerity, form and amplitude, as governed by various parameters such as impounded water level, initial rate of flow downstream from the dam, and river bed ronghness.

\section{INTRODUCTION}

Parmi les problèmes posés par les phénomènes d'intumescences dans les canaux découverts, celui de l'onde de crue consécutive à la lâchure brusque d'un débit a attiré l'attention du Laboratoire d'Hydraulique depuis fort longtemps et l'on sait toute l'importance que peut avoir ce phénomène dans certains cas.

Jusqu'à présent, les études avaient porté sur des cas plus ou moins théoriques et nous avons préféré examiner le problème industriel, c'est-à- dire considérer une vallée de forme assez. sinueuse et de pente relativement élevée.

Dans cette étude, nous nous sommes particulièrement attachés à examiner l'influcnce des paramètres suivants :

--- rugosité du lit,

- tirant d'eau à l'amont du barrage,

sur les caractéristiques de l'onde, en particulier sur la célérité et l'amplitude du front. 


\section{CHAPITRE I}

\section{PROBLĖMES ENVISAGES ET REALISATION DU MODELLE}

\section{Problème envisagé.}

Des études théoriques ou expérimentales ont été réalisées sur la propagation de l'onde de crue subite à l'aval d'un harrage. Quelques solutions graphiques ou mathématiques ont été données à ce problème. Nous ne jugeons pas utile de les rappeler, constatant simplement la complexité du problème qui demeure encore sans solution définitive et complète.

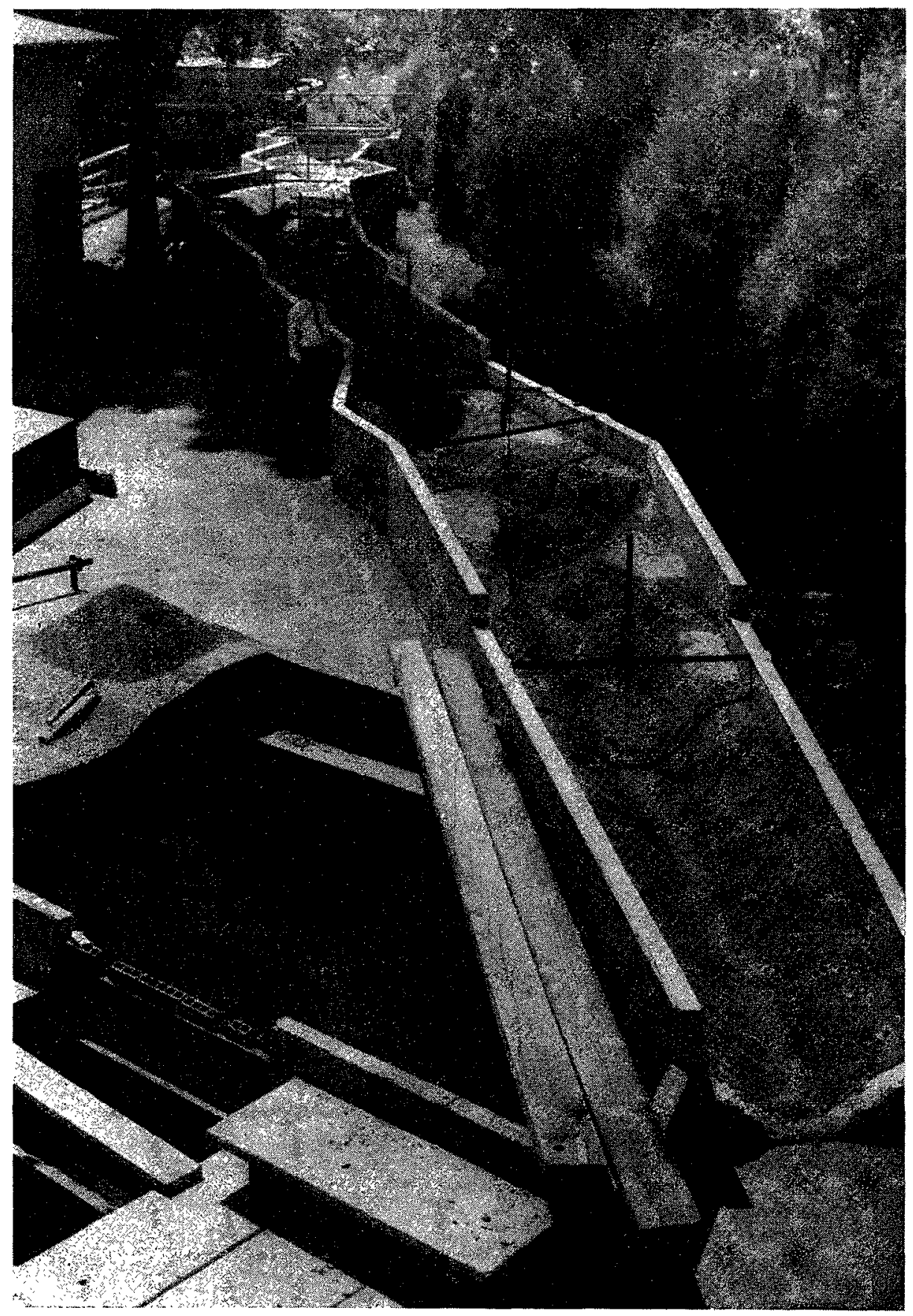

FIci. 1 


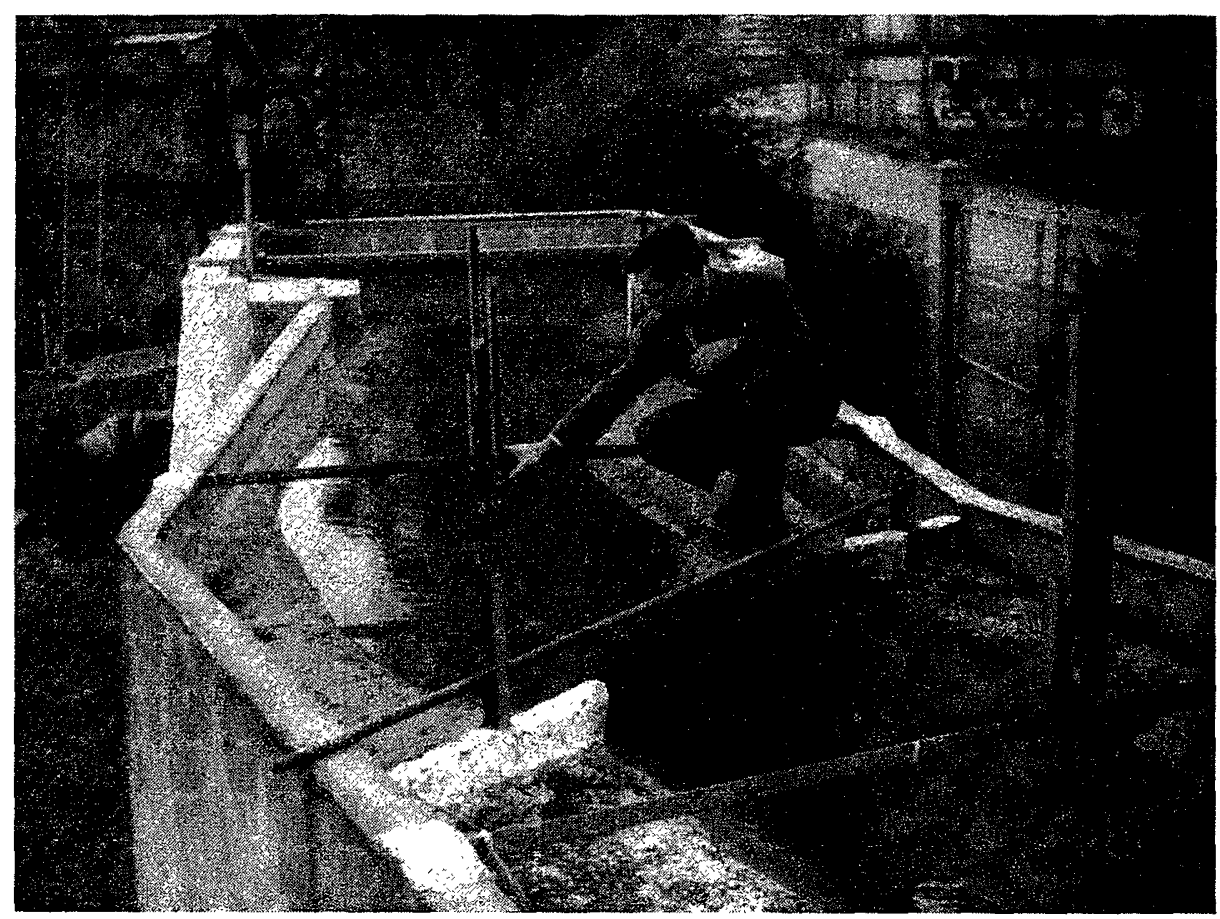

Fifi. 2

Le Laboratoire a entrepris, dans ce cadre, un programme d'études très vaste. Nous avons estimé que, pour mener à bien l'étude, il lui fallait une base expérimentale complète et approfondie dans un cas concret. C'est pourquoi, le Laboratoire a choisi de représenter une vallée existante, en l'espèce la vallée de la Truyère, en aval du barrage de Sarrans.

Nous nous sommes trouvés en présence d'un grand nombre de paramètres (rugosité, pente, section, tirant d'eau en amont du barrage, etc.) et nous avons pensé qu'il fallait systématiquement déterminer leur influence sur le phénomène. Nous avons, en premier lieu, étudié expérimentalement l'influence de la retenue et de la rugosité en considérant :

- plusieurs valeurs de la rugosité;

- dans chacun de ces cas en faisant varier le tirant d'eau amont et le débit initial.

Le présent rapport donne les résultats complets de cette étude.

\section{Réalisation du modèle.}

Nous rappelons brièvement les caractéristiques du modèle construit pour la présente étude et dont la figure 1 donne une vue d'ensemble.

Le modèle réduit, réalisé à l'échelle unique $\lambda=1 / 300$, comprend de l'amont vers l'aval:

- Un bassin d'alimentation à niveau constant;
- Un troncon amont représentant la vallée de la Truyère sur une longueur de $1,6 \mathrm{~km}$ en amont du barrage;

- Le barrage proprement dit;

- Un tronçon aval représentant $12 \mathrm{~km}$ de vallée;

- Un ouvrage de restitution et de mesure du débit.

Un élément important consistait à rendre instantané l'effacement du barrage. Celui-ci a done été constitué par deux tôles en duralumin, chacune articulée sur les flancs de la vallée.

La photographie de la figure 2 montre le barrage et sa retenue ainsi que le bassin d'alimentation.

\section{Système de mesures.}

Nous avons adopté pour la mesure des variations du tirant d'eau, les procédés devenus classiques utilisant les jauges à résistances avec système d'enregistrement approprié.

Nous disposions, au début de l'étude, de huit jauges pour mesurer autant la célérité du front d'onde que les hauteurs d'eau. Nous nous sommes aperçus après les premiers essais que la mise en place de douze jauges, réparties judicieusement le long de la vallée, nous permettrait de mieux étudier la propagation de l'onde, de définir la célérité avec plus de précision et de 


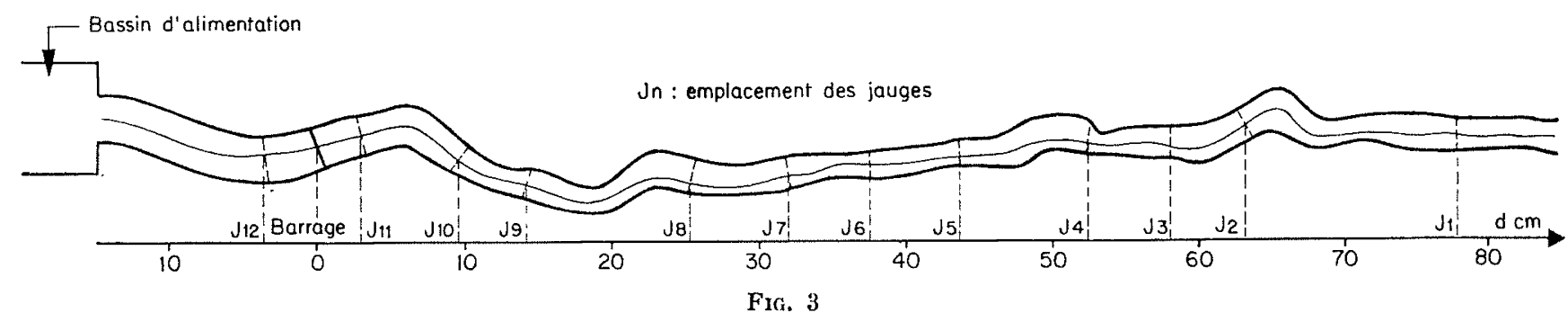

Vue en plan de la vallée.

pouvoir caractériser un coefficient de rugosité, comme nous le verrons plus loin.

La figure 3 permet de situer les jauges sur le modèle. Nous donnons leur distance par rapport au barrage et leur hauteur en cote N.G.F. audessus de l'extrémité aval prise comme plan de référence.

\section{Solution adoptée pour la variation de la rugosité.}

Il importe que l'étude de l'influence de la rugosité puisse être réalisée sans modifier la section de la vallée et la structure du modèle.

Nous avons placé sur le modèle, du bassin d'alimentation à l'extrémité aval, plusieurs séries de grillage en métal déployé, épousant la forme de la vallée et présentant des mailles de différentes dimensions. Ce dispositif pratique et que nous pouvions changer à volonté, nous a permis de poursuivre aisément nos recherches et d'obte- nir un éventail assez large de résultats, ce qui nous a facilité certaines comparaisons.

C'est ainsi que, pour les cinq séries d'essais correspondant à cinq valeurs de la rugosité, nous avons utilisé trois grillages différents :

- Série I : sans grillage;

- Série II : grillage de mailles de $115 \mathrm{~mm}$ sur 40 ;

- Série III : grillage de mailles de $65 \mathrm{~mm}$ sur 25;

\section{Série IV :} grillages de mailles de $200 \mathrm{~mm}$ sur 175 ;

- Série V : superposition des grillages de la série III et de la série IV.

La photographie de la figure 4 permet de voir l'un des grillages installés sur le modèle et l'on peut se rendre compte qu'il épouse parfaitement le profil de la vallée.

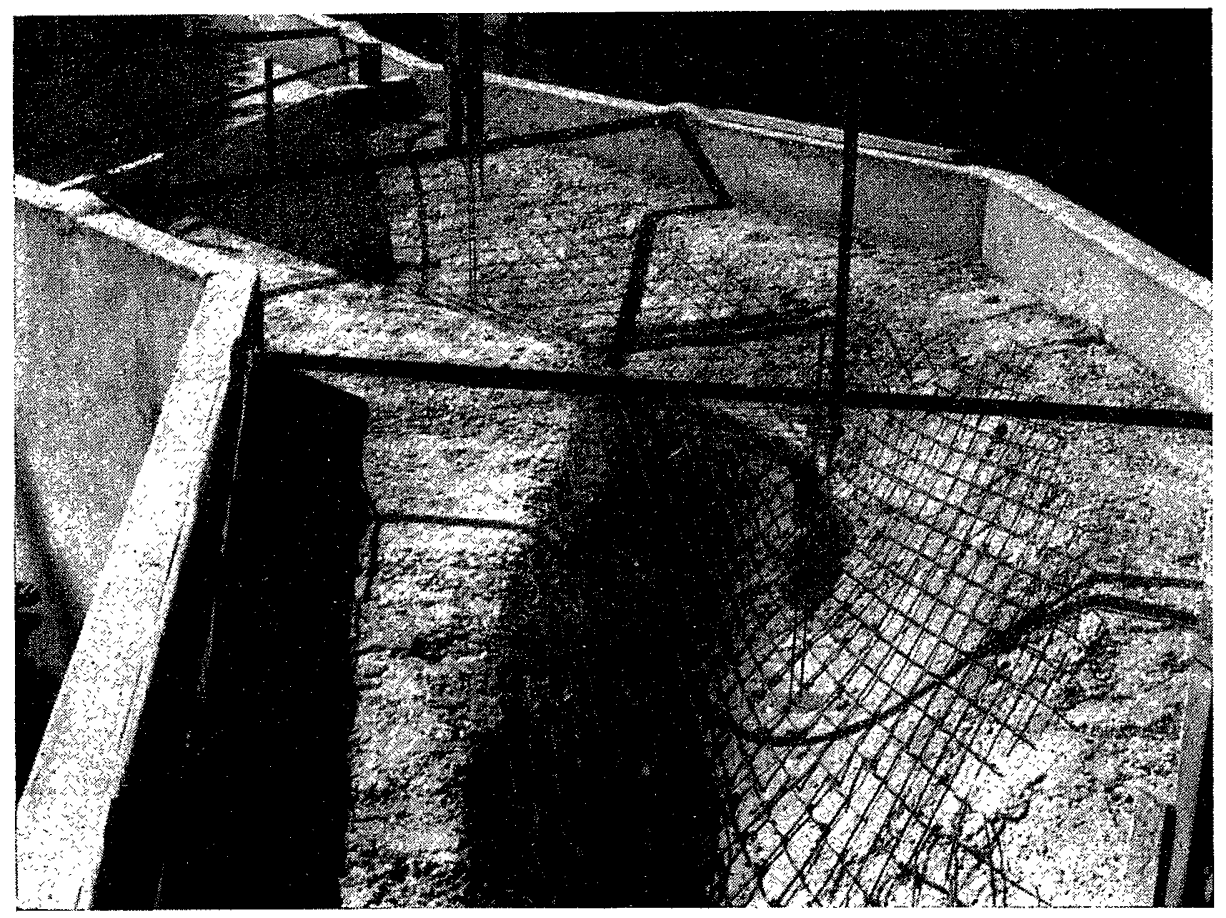

FIG. 4 


\section{CHAPITRE II}

\section{REALISATION DES EXPERIENCES}

Toutes les expériences sont effectuées de manière identique, la rugosité variant pour chacune d'elles. C'est ainsi que, pour chaque série, nous opérons pour trois valeurs de la retenue en amont du barrage avec un débit initial nul et deux valeurs de la retenue en amont avec un débit initial correspondant à une crue moyenne et à une crue maximale dans la vallée.

Une expérience comprend essentiellement deux phases:

- l'étalonnage;

- l'expérience proprement dite.

\section{$1^{\circ}$ L'étalonnage :}

Afin d'éliminer l'influence de la résistivité de l'eau, nous avons été amenés à procéder à un étalonnage avant chaque essai. La durée d'un étalonnage et d'un essai étant d'environ deux heures, la variation de la résistivité est, dans ce cas, négligeable comme l'ont montré de nombreuses expériences antérieures. Cette méthode est peut-être longue et fastidieuse, mais l'installation d'un système éliminant la résistivité, en raison du nombre de jauges utilisées, aurait malheureusement compliqué les dispositions expérimentales.

De plus, ayant constaté, en régime permanent, la présence d'un sillage aéré derrière les fils des jauges, les étalonnages ont été réalisés non à l'état statique, mais dans l'état dynamique. Nous avons installé ainsi, près de chacune des jauges, une pointe de mesure qui nous permel, en régime permanent, de repérer la position du plan d'eau.

Etant donné la linéarité de la variation de la hauteur en fonction de la dérivation, nous avons déterminé cinq à six points de la courbe d'étalonnage correspondant à cinq ou six régimes permanents pour un débit variant de

\section{0 à $14.10^{4} \mathrm{~m}^{3} / \mathrm{s}$,}

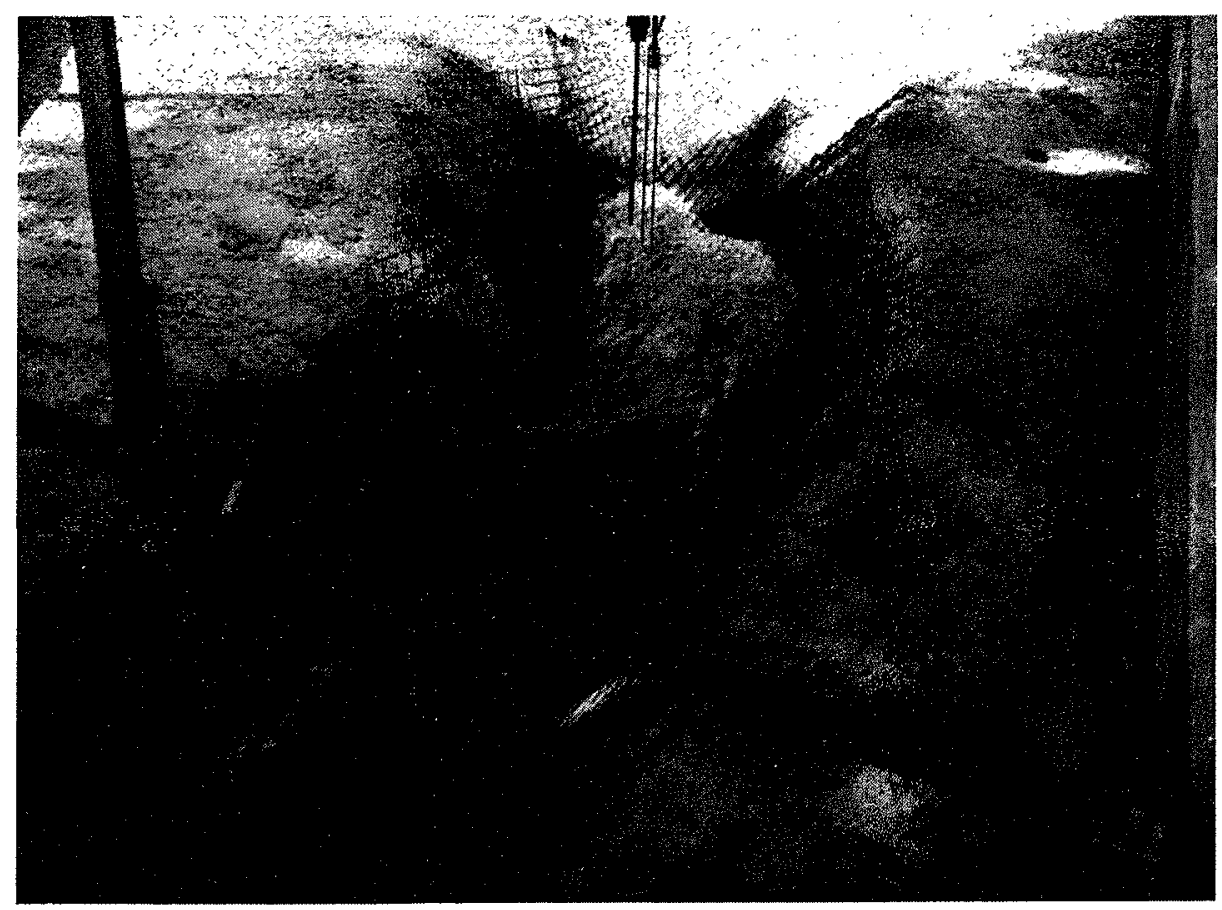

FIG. :) 


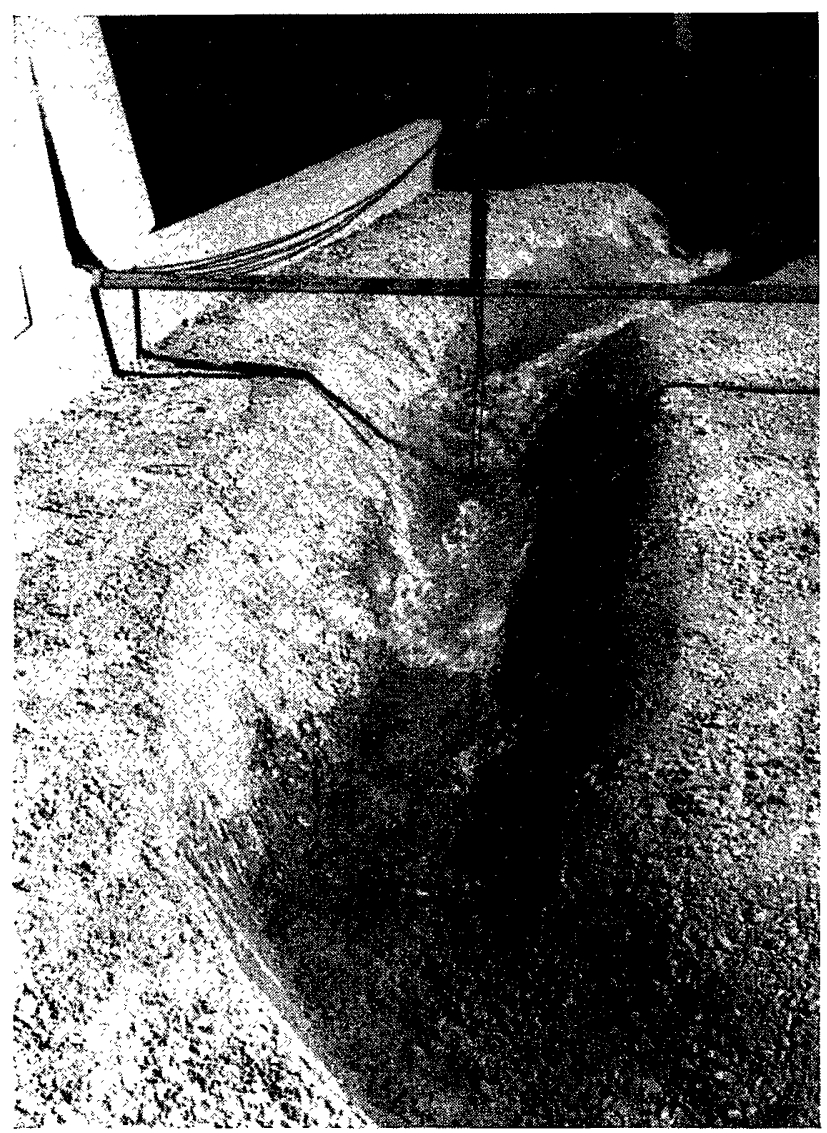

FII. 6

grandeur ouvrage. Nous avons enregistré ces valeurs sur le papier photographique pour les douze jauges, ce qui nous a permis d'établir les courbes d'étalonnage pour chacune d'elles, connaissant d'une part les hauteurs relatives à chaque jauge que nous déterminions avec les pointes de mesure, et d'autre part, les déviations enregistrées.

\section{$2^{\circ}$ L'expérience proprement dite :}

L'expérience consiste essentiellement dans l'enregistrement des tirants d'eau dans les profils en travers où sont situées les jauges, en fonc-

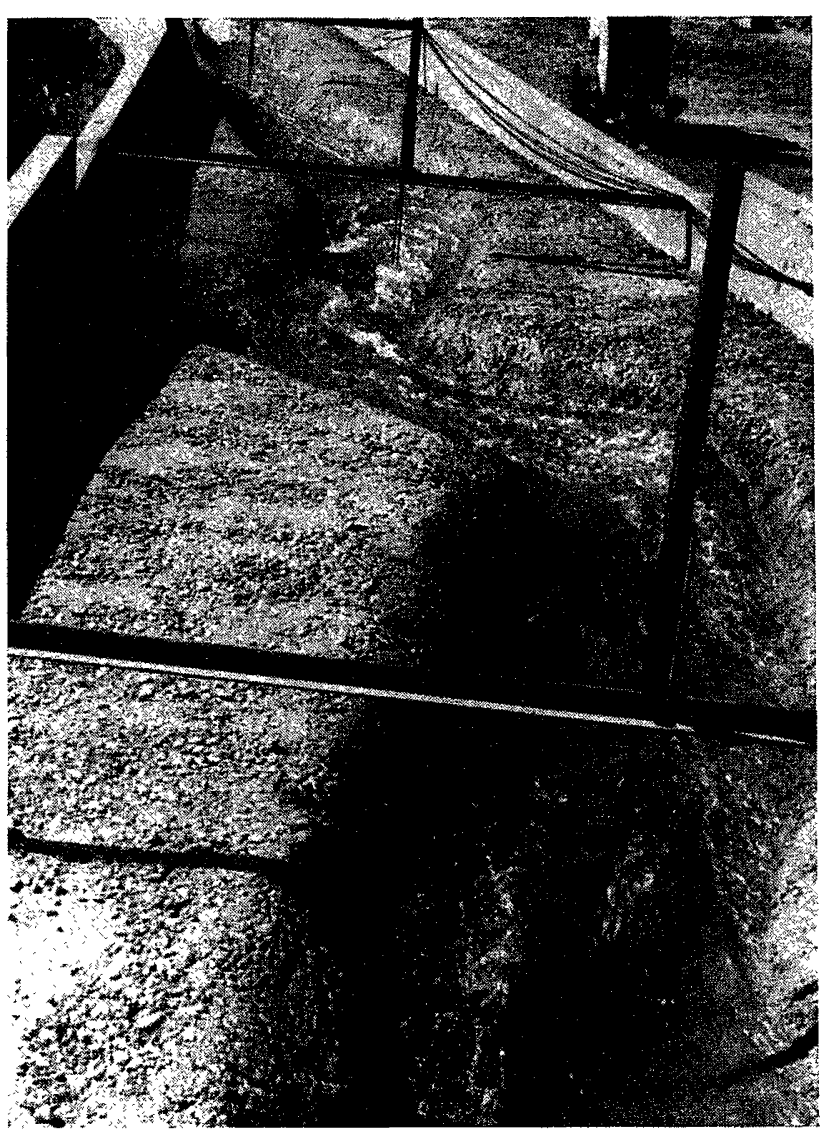

Fig. 7

tion du temps. Ces enregistrements nous ont permis de déterminer :

- L la loi $h(t)$ en certains points de la vallée;

- le profil de la surface libre à un instant $t$;

- la célérité de l'onde.

Aussitôt l'étalonnage terminé, nous établissions dans la vallée, un régime permanent correspondant aux conditions aval prévues par l'expérience. Nous pouvions alors réaliser l'enregistrement.

Les photographies des figures 5,6 et 7 montrent la propagation du front d'onde le iong de la vallée dans différents cas. 


\section{CHAPITRE III}

\section{RESULTATS}

\section{Notations utilisées.}

$h$ tirant d'eau;

$\mathrm{H}_{0}$ cote du fond de la vallée par rapport à un plan horizontal de référence (extrémité aval du modèle);

$t$ temps compté à partir de la rupture du barrage;

$\mathrm{X}$ abscisse comptée à partir du barrage;

$\mathrm{V}$ vitesse de déplacement du front d'onde;

$a$ célérité propre du front d'onde;

$\mathrm{U}_{0}$ vitesse moyenne dans la vallẻe;

$Q$ débit;

$k_{r}$ coefficient d'écoulement;

I pente du fond de la vallée.

\section{Détermination du paramètre caractérisant la rugosité.}

\section{1) Position du problème :}

Nous avons cherché à déterminer un coefficient représentant l'état réel du modèle qui nous aurait permis de classer les différentes rugosités que nous avons étudiées dans l'une ou l'autre catégorie définissant la nature des parois. En général, ce coefficient est calculé par les formules classiques et en particulier celles de Manning ou Strickler, lorsque nous sommes en présence d'une vallée uniforme dont l'écoulement à un mouvement uniforme. Mais le modèle représente une vallée sinueuse, de section variable et dont la pente élevée ( $3 \%$ environ) nous donne un régime torrentiel. Ceci nous a amené à définir conventionnellement un coefficient représentant la rugosité dans le cas de notre étude : nous le désignerons par la suite par le terme «coefficient d'écoulement». Ce coefficient tient compte de la rugosité propre des parois, des pertes de charge singulières dues à l'irrégularité des profils, à la sinuosité du lit, etc.

2) Détermination du coefficient d'écoulement:

La détermination du coefficient pour les cinq séries d'essais a été faite sur la base de la formule de Strickler

$$
k_{v}=\frac{\mathrm{V}}{\mathrm{R}^{3 / 3} \mathrm{I}^{1 / 2}}
$$

en considérant un écoulement permanent en régime uniforme dans la vallée.

Nous avons procédé aux relevés des profils en long de la surface libre en régime permanent pour plusieurs valeurs du débit $Q$. Le débit de régime permanent correspondant au régime uniforme, n'a pu être déterminé avec grande précision. Pour chacun des débits réalisés, nous avons noté les tirants d'eau en trois sections transversales de la vallée situées sur des parties rectilignes (troncon de la vallée compris entre la jauge 8 et la jauge 4). Nous avons calculé le rayon hydraulique $R$ et la vitesse $V$ dans chacun des cas, ce qui nous a donné $k_{r}$.

Nous précisons les valeurs moyennes du coefficient d'écoulement, $k_{r}$, retenu pour caractériser chaque série d'essais.

$$
\begin{array}{ll}
\text { - Série I : } & k_{r}=58,40 \\
\text { - Série II : } & k_{r}=40,00 \\
\text { - Série III : } & k_{r}=38,80 \\
\text { - Série IV : } & k_{r}=36,60 \\
\text { - Série V : } & k_{r}=32,60
\end{array}
$$

\section{Résultats expérimentaux.}

Pour étudier le comportement de l'onde, au cours de sa propagation dans la vallée, nous avons effectué diverses expériences, chacune d'elles ayant été réalisée pour les rugosités que nous avons envisagées.

Ces essais ont été faits avec les conditions suivantes :

- Retenue pleine, débit initial nul;

- Retenue aux 2/3 pleine, débit initial nul;

- Retenue au $1 / 3$ pleine, débit initial nul;

- Retenue pleine, débit initial égal à $780 \mathrm{~m}^{3} / \mathrm{s}$ (crue moyenne);

- Retenue pleine, débit initial égal à $2100 \mathrm{~m} 3 / \mathrm{s}$ (crue maximale).

Les tableaux reproduits en annexe donnent les valeurs de la célérité du front d'onde déterminées pour chaque valeur de rugosité entre jauges successives. 


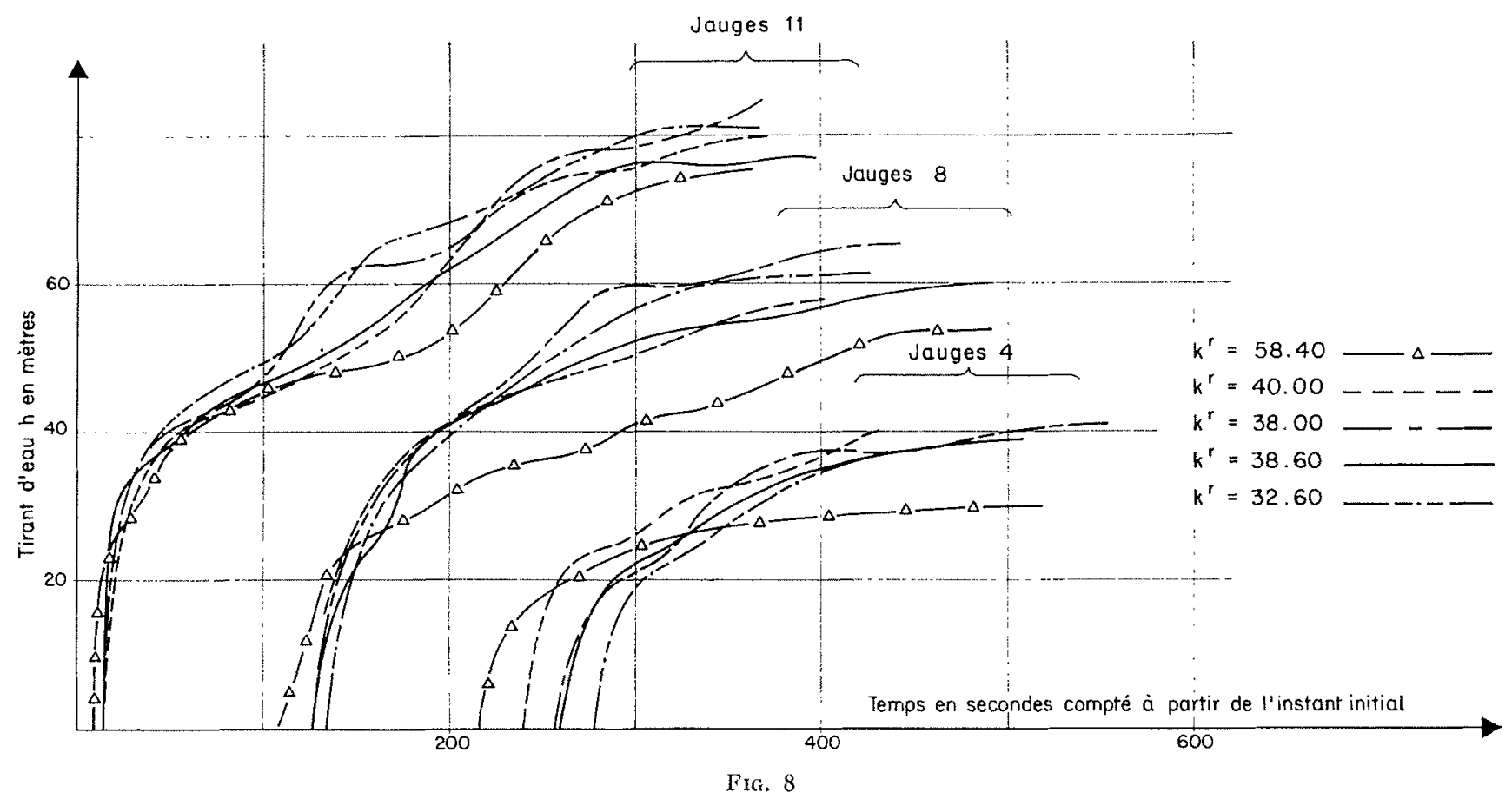

Courbes $h(t)$ aux profils 11,8 et 4 . Retente pleine. Débit initial nul.

En outre, les enregistrements que nous avons réalisés nous ont permis de tracer la forme et l'évolution $\mathrm{du}$ front d'onde à différentes jauges pour les cinq séries d'expériences envisagées. Les figures $n^{\circ} 8$ et 9 fournissent un de ces enregistrements.

L'examen de ces résultats permet de montrer quelle est l'influence de divers paramètres.

\section{Influence de la rugosité.}

Les tableaux donnant les valeurs de célérité, suivant les valeurs de la rugosité, permettent d'énoncer les résultats essentiels suivants :

a) La variation de la célérité moyenne du front d'onde pour toutes les retenues envisagées

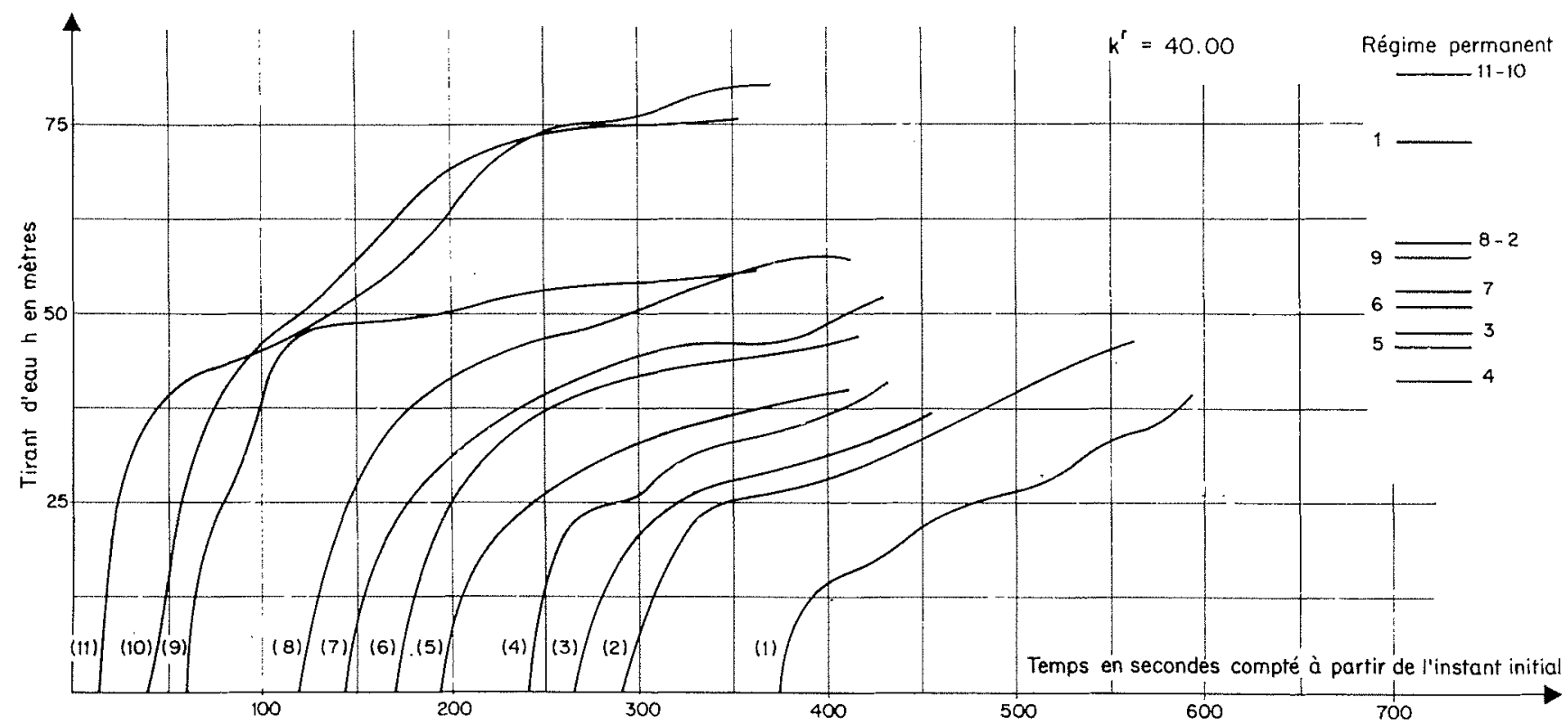

FIG. 9

Courbes $h(t)$ en différents profils. Retenue pleine. Aval sec. 
est d'environ $30 \%$ en considérant les valeurs extrêmes de $k_{r}$. Cette variation est du même ordre en tous les points de la vallée, sauf dans le troncon situé immédiatement à l'aval du barrage, où elle est très faible.

b) La forme du front d'onde est pratiquement la même au cours des premiers instants de la propagation, e'est-à-dire dans la première partie de la vallée, à l'aval du barrage. On note toutefois et par la suite, certaines variations qui demeurent assez faibles par rapport au tirant d'eau considéré. On peut donc préciser que la variation de l'amplitude du front d'onde est peu influencée par la valeur de la rugosité.

\section{Influence de la retenue amont et du débit initial avant la rupture.}

Les quelques conclusions suivantes peuvent être déduites des tableaux résumant les expériences.

a) Si l'on compare la valeur de la célérité moyenne pour une même valeur de la rugosité, et pour les hauteurs de retenue $h$ différentes à l'amont on constate que cette valeur moyenne décrô̂t en même temps que la hauteur $h$ : ce résultat était évidemment prévisible.

b) Si l'on compare les résultats avec débit nul puis avec débit initial, on voit que la célérité est plus importante dans le cas où il existe un débit initial. Cet accroissement de la vitesse de propagation semble donc suivre la loi $\mathrm{V}=a+u_{0}$ sauf, peut-être, aux premiers instants de la propagation. Ce résultat est important, car il permet, sans nul doute, d'envisager pour l'étude de l'onde de crue une généralisation de la théoric des intumescences.

c) $\mathrm{Si}$ le front d'onde demeure raide, quelle que soit la valeur de la hauteur $h$ de la retenue, son amplitude décroìt en même temps que cette hauteur de retenue.

Ces conclusions sont donc particulièrement importantes, puisqu'elles permettent de voir la nette influence de la retenue à l'amont du barrage avant l'effacement de celui-ci.

\section{CONCLUSION}

L'étude expérimentale de la propagation d'une onde de crue, effectuée comme nous venons de le préciser, nous a permis d'aboutir à quelques conclusions importantes. Nous rappelons que :

- Le modèle réduit représente une vallée existante dont la pente moyenne est d'environ $3 \%$. L'étude a donc été faite à partir d'un cas concret;

- Les différents coefficients de rugosité, que nous avons appelés « coefficients d'écoulement» n'ont été déterminés que dans le but de pouvoir classifier et comparer les résultats obtenus.

L'essentiel de ces conclusions peut se résumer ainsi :

1. Sans préjuger de l'influence de la pente du lit, mais considérant la variation de la célérité du front d'onde sur chaque troncon par rapport à la célérité moyenne, nous estimons, dans le cadre de notre étude, que le front d'onde se propage dans la vallée avec la même vitesse. Le front demeure raide, mais son amplitude va en décroissant.

2. La hauteur de retenue à l'amont du barrage et un débit initial ont une influence déterminante sur l'onde. Nous avons observé que la célérité et l'amplitude décroissaient en même temps que la hauteur $h$ de la retenue à l'amont avant l'effacement. D'autre part, la vitesse du front d'onde, en présence d'un débil initial, semble suivre la loi classique de célérité des intumescences.

3. On aurait pu penser, a priori, que la rugosité ait une influence notable tant sur la célérité que sur l'amplitude de l'onde. Or, cette influence, comme nous venons de le voir, est relativement peu importante. 
ANNEXE

CÉLÉRITÉ DU FRONT D'ONDE EN $\mathrm{m}^{3} / \mathrm{s}$

\begin{tabular}{|c|c|c|c|c|c|c|c|}
\hline \multicolumn{8}{|c|}{ RETENUE PLEINE - DÉBIT INITIAL NUL } \\
\hline Jauges & 11 à 9 & 9 à 8 & 8 à 6 & 6 à 4 & 4 à 2 & 2 à 1 & $11 \dot{a} 1$ \\
\hline$k_{r}=58,40 \ldots \ldots \ldots$ & 34,7 & 30,07 & 30,4 & 26,9 & 33,8 & 25,26 & 29,67 \\
\hline$k_{r}=40,00 \ldots \ldots \ldots$ & 33,40 & 26,58 & 29,15 & 23,10 & 31,75 & 22,60 & 26,98 \\
\hline$k_{r}=38,00 \ldots \ldots$ & 31,98 & 25,05 & 25,46 & 22,27 & 27,28 & 22,08 & 25,07 \\
\hline$k_{r}=36,60 \ldots \ldots \ldots$ & 31,41 & 25,40 & 24,79 & 20,82 & 28,70 & 20,42 & 24,54 \\
\hline$k_{r}=32,60 . . \cdots \cdots$ & 27,21 & 24,30 & 23,22 & 20,58 & 25,40 & 19,87 & 22,99 \\
\hline \multicolumn{8}{|c|}{ RETENUE $2 / 3$ PLEINE - DÉBIT INITIAL NUL } \\
\hline$k_{r}=58,40 \ldots \ldots \ldots$ & 32 & 25,93 & 25,9 & 23,7 & 30,5 & 22,37 & 26,15 \\
\hline$k_{r}=40,00 \ldots \ldots \ldots$ & 28,03 & 24,38 & 23,05 & 19,98 & 26,68 & 18,78 & 22,48 \\
\hline$k_{r}=38,00 . \ldots \ldots \ldots$ & 28,87 & 22,02 & 22,93 & 19,85 & 25,78 & 19,18 & 22,58 \\
\hline$k_{r}=36,60 \ldots \ldots \ldots$ & 26,40 & 20,58 & 20,08 & 17,91 & 24,08 & 16,18 & 19,92 \\
\hline$k_{r}=32,60, \ldots \ldots \ldots$ & 23,60 & 18,73 & 19,26 & 16,98 & 21,41 & 16,53 & 18,98 \\
\hline \multicolumn{8}{|c|}{ REtenUe $1 / 3$ PLEINE - DÉBit INItTal NUL } \\
\hline$k_{r}=58,40 \ldots \ldots \ldots$ & 26,1 & 21,83 & 21,4 & 19,9 & 21,6 & 18,21 & 21,71 \\
\hline$k_{r}=40,00 \ldots \ldots \ldots$ & 21,96 & 17,55 & 18,508 & 15,63 & 21,33 & 15,03 & 17,82 \\
\hline$k_{r}=38,00 . \ldots \ldots \ldots$ & 19,51 & 14,71 & 14,92 & 14,20 & 17,55 & 13,18 & 15,32 \\
\hline$k_{r}=36,60 . \ldots \ldots \ldots$ & 22,60 & 16,98 & 16,96 & 14,76 & 19,65 & 13,87 & 16,81 \\
\hline$k_{r}=32,60 . \cdots \cdots \cdots$ & 16,18 & 13,59 & 14,08 & 12.71 & 15,90 & 12,11 & 13,86 \\
\hline \multicolumn{8}{|c|}{ RETENUE PLEINE - DÉBIT INITIAL $780 \mathrm{~m}^{3} / \mathrm{s}$} \\
\hline$k_{r}=58,40 . \ldots \ldots \ldots$ & 34,7 & 33,02 & 31,9 & 28,00 & 35,2 & 26,14 & 30,89 \\
\hline$k_{r}=40 ; 00 \ldots \ldots \ldots$ & 31,45 & 30,39 & 28,80 & 25,70 & 31,30 & 23,13 & 27,90 \\
\hline$k_{r}=38,00 . \ldots \ldots \ldots$ & 31,18 & 28,18 & 25,50 & 23,60 & 27,70 & 21,65 & 25,75 \\
\hline$k_{r}=36,60 \ldots \ldots \ldots$ & 31,18 & 29,62 & 26,65 & 28,05 & 29,82 & 21,32 & 26,42 \\
\hline$k_{r}=32,60 . \cdots \ldots \ldots$ & 29,65 & 26,40 & 24,40 & 22,40 & 25,89 & 19,86 & 24,12 \\
\hline \multicolumn{8}{|c|}{ RETENUE PLEINE - DÉBIT INITIAL $2100 \mathrm{~m} \% / \mathrm{s}$} \\
\hline$k_{r}=58,40 . \ldots \ldots$ & 33,1 & 35,95 & 33,1 & 29,1 & 36,5 & 31,73 & 32,0 \\
\hline$k_{r}=40,00 . \ldots \ldots \ldots$ & 31,50 & 33,62 & 30,05 & 27,60 & 32,78 & 24,35 & 29,40 \\
\hline$k_{r}=38,00 . \ldots \ldots \ldots$ & 31,41 & 30,50 & 27,42 & 25,00 & 28,70 & 22,50 & 26,98 \\
\hline$k_{r}=36,60 \ldots \ldots \ldots$ & 31,30 & 30,27 & 27,60 & 28,41 & 30,95 & 21,62 & 26,92 \\
\hline$k_{r}=32,60 \ldots \ldots$ & 30,70 & 28,48 & 25,85 & 23,90 & 26,70 & 20,10 & 25,10 \\
\hline
\end{tabular}




\section{I S C U S I O N}

Président : М. Chapolthien

M. le Président remercie M. le Professeur Novgano pour son exposé très clair et lui pose deux questions : - Dans une similitude de pesanteur, la transposition du coefficient $K_{r}$ en vraie grandeur, ne pose-t-elle pas de problème?

- Au sujet du front d'in'umescence, est-ce la célérité (ie la crête du début ou la célérité moyenne qui a été mentionnée?

II. Novgaro répond à la première question que le problème serait identique à celui qui se poserait pour la transposition du coefficient de strickler et qu'il n'y a pas d'inconvénient à le faire. Celui que l'on peut avoir dans la transposition du coefficient $K r$ est minimisé vis-à-vis de la schématisation que l'on fait sur le modèle réduit.

A la deuxième question, M. Novgaro répond qu'il a mentionné la célérité de la crète du début, c'est-à-dire le moment rigoureux où l'intumescence commence à atteindre la jauge de la résistance. La variation de la célérité ne dépasse pas $5 \%$, en considérant la célérité moyenne.

M. Rodier demande quelles sont les vitesses mesurées pour retenue pleine après transposition dans la nature.

M. Nougaro précise qu'il a obtenu des célérités de lordre de $100 \mathrm{~km} / \mathrm{h}$ et des hauteurs atteignant 50 ì $60 \mathrm{~m}$, valeurs transposées à l'ouvrage.

M. Remenieras montre, en s'inspirant des méthodes graphiques dérivées de celle de M. Bengenon, que dans un canal d'amenée revêtu en béton et avec les vitesses usuelles, la variation de la célérité avec la rugosité du canal est assez faible. Il est remarquable que dans les essais exposés par $M$. Nougaro, cette influence de la rugosité reste faible malgré les vitesses élevées et les rugosités fortes réalisées.

M. Novgano ajoute que Calame a donnć pour les canax une relation qui, simplifiéc, serait:

$$
\sqrt{\frac{g \mathrm{H}}{1+f(\mathrm{~S})}} ;
$$

cette fonction de la perte de charge étant extensive même pour des variations importantes du coefficient de rugosité. Cetle onde de crue donne des résultats se rapportant à ceux des canaux.

M. REMENIÉRAS pense que pour des raisons économiques, les modèles utilisés pour l'étude de la progagation des intumescences sur de longs secteurs de cours d'eau devront etre « distordus »; mais en raison de l'incertitude qui règne encore sur la représentation correcte de fortes oscillations secondaires avec ce genre de modèle, il est logique d'expérimenter d'abord sur modèles non distordus, ainsi que l'a fail M. Novgano. Il souligne qu'un modèle trop court ne permet pas d'étudier l'ćrolution de l'intumescence (même dans une section relatirement proche du barrage) sur une durée suffisante; cela est dû̀ à l'impossibilité de réaliser audelà d'un certain instant, des conditions correctes aux limites aval du modèle.

Sur la demande de ir. Chamuleat, M. Notgaho précise que la pente du modèle correspond exactement à la pente de la vallée et qu'elle est assez faible.

D'après une question de M. Ferny, M. Novgano dit que le tracé de la rivière est limité a $12 \mathrm{~km}$ de Sarrans, ce qui correspond à psu près à la restitution de Brommat.

M. Fenry dit qu'ainsi on peut se faire une idée de la pente, la chute de Brommat etant d'environ $250 \mathrm{~m}$. La pente est donc ćgale à environ $2 \%$.

M. Novgano, d'après une remarque de Mi. Preissmaxs, précise que les $3 \%$ qu'il a cités dans son étude ne sont valables que pour certaines sections ayant une pente relativement élevée, ce qui a empêchè de schématiser ce mouvement uniforme el de déterminer un coefficient exact de rugosité

M. Lazard demande si l'on connaît le lieu des maximums à partir du barrage pour efudier le cas où il se rompt brusquement of savoir quels sont les dangers prósentés par l'onde d'intumescence.

M. Novgaro répond qu'il n'en a pas parlé dans son rapport mais qu'il l'a montré sur les clichés. La hauteur d'eau, qui variait, est de l'ordre de $60 \mathrm{~m}$. Pour le cas où la retenue est pleine et le débit initial nul, le point optimal représentait $70 \mathrm{~m}$ au droit du barrage dans la vallée. Au bout de $12 \mathrm{~km}$, il y avait $40 \mathrm{~m}$. Le premier problème posé étant consécutif à des soucis d'arertissement des populations, ce qui etait, a priori, intéressant, était la célérité et non la hateur d'eau.

M. Kolkman indique que londe subite est comparable au phénomène de l'échange de deux liquides arec des densites differentes. An Laboratoire Hydraulique de Delft, on a constaté qu'il n'y a pas de décélération d'un coin salé avant qu'un trajet de 100 fois la profondeur soit parcouru. Cet essai est fait avec une hauteur du coin de $0,1 \mathrm{~m}$ et une différence de densilé d'environ $15 \%$. On peut dire que les observations que M. Nougaro a montrées sont en concordance avec les essais avec le coin salé. Che\% les derniers une influence plus grande du frottement est possible.

M. Ie Président remercie M. Konkmas pour son intervention et M. Novgano pour son exposé. 